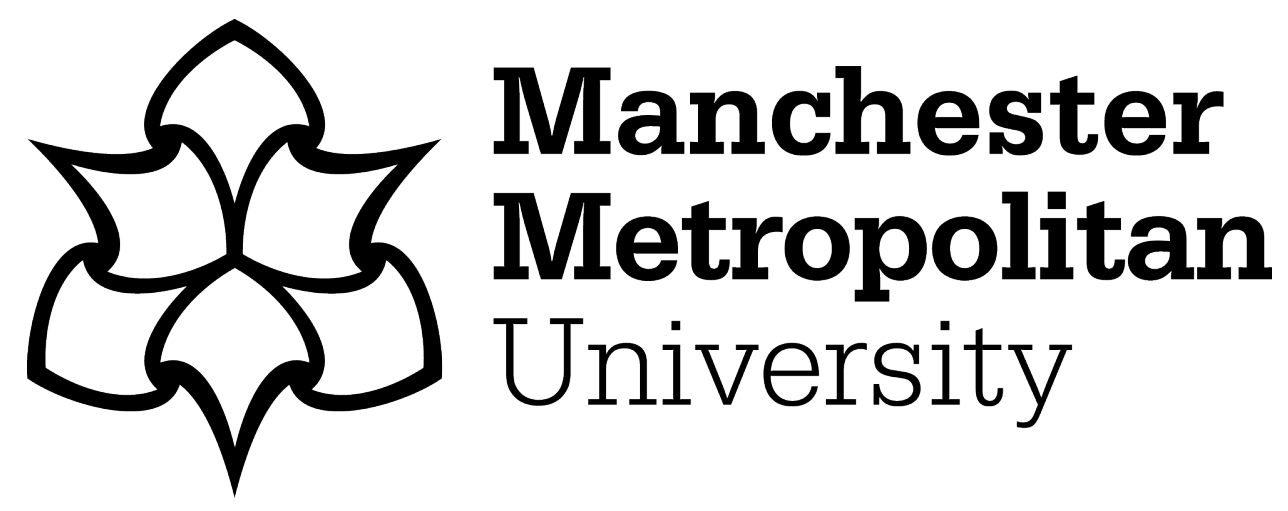

Jayaraman, R, Raja, G, Bashir, AK, Hussain, CS, Hassan, A and Alqarni, MA (2018) Interference Mitigation Based on Radio Aware Channel Assignment for Wireless Mesh Networks. Wireless Personal Communications, 101. pp. 1539-1557. ISSN 0929-6212

Downloaded from: https://e-space.mmu.ac.uk/622912/

Version: Accepted Version

Publisher: Springer Verlag

DOI: https://doi.org/10.1007/s11277-018-5776-4

Please cite the published version 


\title{
Interference Mitigation Based on Radio Aware Channel Assignment for Wireless Mesh Networks
}

\author{
Ramkumar Jayaraman ${ }^{1}$, Gunasekaran Raja ${ }^{1}$, Ali Kashif Bashir ${ }^{2}$, Chauhdary Sajjad \\ Hussain ${ }^{3}$, *Ali Hassan ${ }^{3}$, Mohammed A. Alqarni ${ }^{3}$ \\ ${ }^{1}$ Department of Computer Technology, Anna University, Chennai, India \\ ${ }^{2}$ Department of Science and Technology, University of the Faroe Islands, Faroe Islands, Denmark \\ ${ }^{3}$ Faculty of Computing and Information Technology, University of Jeddah, \\ P. O. Box 34, Jeddah 21959, Saudi Arabia \\ Tel: +966-126952000 Ext. 74237 \\ *Corresponding Author Email: ahsssan1@uj.edu.sa
}

\begin{abstract}
An intricate network deployment for high demand users leads to simultaneous transmission in wireless mesh networks. Multiple radios are adapted to individual nodes for improving network performance and Quality of Service (QoS). However, whenever multiple radios are assigned to the same channel, co-located radio interference occurs, which poses a major drawback. This paper proposes a Radio aware Channel Assignment (Ra-CA) mechanism based on a direct graphical model for mitigation of interference in multi-radio multi-channel networks. Initially, the co-located radio interference is identified by classifying non-interfering links for simultaneous transmission in the network. Proposed channel assignment mechanism helps in allocating the minimal number of channels to the network that mitigate co-located radio interference. Performance analysis of the proposed Ra-CA strategy is carried out compared with other existing techniques, like BFS-CA and MaIS-CA, in multi-radio networks. Simulation results demonstrate that the proposed channel assignment scheme is more efficient compared to the existing ones, in terms of QoS parameters like, packet drop rate, packet delivery ratio, transmission delay and throughput.
\end{abstract}

Keywords: Multiple-radio, Channel Assignment, Co-located Radio Interference, Mesh Networks and QoS

\section{Introduction}

Wireless Mesh Networks (WMNs) [1] support multiple hop connectivity with self-organized, self-configured nodes, which can also act as routers to extend network coverage area. WMN [2] [3] plays a promising role to provide continuous connectivity to the users in a wireless network environment. Recent wireless networking technologies strive to connect more users using surplus network resources and to provide seamless communication. Next generation networks such as Long Term Evolution - Advanced (LTE-A) [4], with channel allocation range from 2-3 GHz band, employs Orthogonal Frequency Division Multiplexing (OFDM) to improve network data service 
provisioning. OFDM supports assignment of multiple, closely-spaced, orthogonal sub-carrier radios to an individual node. This capability improves bandwidth provisioning to individual users by enabling simulationeous transmission of data streams, but at the same time suffers from the problem of co-located interference.

The two main categories of interference, namely external and internal interferences, are elaborated in [5] [6]. External wireless devices, such as Bluetooth, which communicate over the same frequency, lead to external interference of uncontrolled activities in the network [7]. During various processes within the network, such as network deployment, resource allocation, routing and scheduling schemes, the communications between neighboring nodes over same frequency could lead to internal interference [8]. Usually multiple radios are placed in a single node for simultaneous transmissions and to provide reliable data services in the network. When multiple radios use same channel or timeslot for data transmission, co-located radio interference could occur. In case of WMNs, where frequent interferences could originate between neighboring nodes, an efficient channel allocation scheme offers a significant impact on the performance of the network. The underlying interference problems also lead to network disruption during establishment of multiple connections and degrade QoS. Various Channel Assignment (CA) schemes [9] [10] have contributed for identifying and mitigating the interferences in WMN. The conflict graph concept is commonly employed to address interference issues in WMN. For MultiRadio Multi-Channel (MRMC) networks, the conflict graph concept can be extended as MRMC conflict graph [11] [12]. The conflict graph approach to multi-radio generation using improvised vertex coloring algorithm is modeled in [11]. The works proposed in [24] [33] [34] discuss the problems of interference related to multi-radio multi-channel networks. However, to the best of our knowledge, existing CA schemes cannot achieve optimal network state based on the number of channels required for the MRMC network.

The focus of this work is to propose Radio aware Channel Assignment (Ra-CA) strategy, which can minimize required number of channels in case of a complex multi-radio network. This study assumes a centralized environment where Base Station (BS) acts as a central entity and maintains all the activities of the Subscriber Stations (SSs). Hereafter, SS is referred to as mobile equipment or device and BS acts as a mobile tower, which monitors and controls activities of one or more SS's.

The proposed scheme attempts to mitigate co-located radio interference problems related to MRMC networks by adopting different channels using CA strategy. The Ra-CA strategy incorporates an independent set algorithm and a two-folded graphical algorithm, which guides it to determine interference links and to assign minimum number of channels required in the network. Subsequently, the requirement of minimal channels in a multi-hope network help to reduce network control traffic and transmission delay.

The main contributions of this work are as follows:

i. This study employs network topology to classify interfering and non-interfering links.

ii. A channel assignment strategy, hereby referred as Ra-CA, is proposed that helps to mitigate interference and to calculate a minimal number of channels required for an improved QoS provisioning in the network. 
The rest of the paper is organized as follows: The discussion on related work is given in section 2 . In section 3, the problem of co-located radio interference is defined for WMN. Section 4 details the proposed Ra-CA strategy using direct graphical method with minimal channel utilization. The proposed Ra-CA strategy is analyzed and evaluated in Section 5. Section 6 of this paper states the conclusion.

\section{Related Work}

Future networking technologies promise to offer high-speed wireless networks with seamless connectivity. However, they also face technically challenging problems like interference [13]. Interference plays a major role in degrading network performance metrics like throughput, scalability and latency in the network. Several types of interferences [35-37] could occur during dense node deployment and synchronization. Channel assignment, routing and scheduling techniques play a vital role to mitigate interference problems in wireless networks [9] [10]. To address the problem of interference, interference aware approach is proposed in [14], which helps to reduce interference by assigning the blocking metric, i.e., number of interfering nodes at the intermediate and improving throughput in WMN [14].

Generally, when a transceiver radio of any node tries to perform more than one transmission and reception in a single time slot, primary interference occurs. When a receiver is located within communication range of more than one sending transceiver during the same time slot, secondary interference occurs. Interference can also be categorized into primary and secondary interferences as explained in [15]. To address the problem of primary and secondary interferences, modified channgel assignment (CA) strategies [16] [38] can effectively be deployed. For WMN networks, Clique Partitioning (CP) technique based on channel assignment is proposed to mitigate the interference problems in MRMC networks [15]. The interference graph is formed by classifying different links to provide a minimal number of channels. Channel assignment, scheduling and routing problems are NP-complete and considered challenging issues for MRMC networks.

Overhearing is a phenomemon that occurs when sensor nodes can hear each other's communication and thus subsequently cause interference. In order to resolve overhearing (also known as listening) interference problem, joint CA and routing approach in the multi-hop network is proposed in [16]. Routing is replaced with network coding as it allows nodes to randomly mix packets during overhearing, prior to forwarding them. A time partitioning approach, specific to address primary interference, is proposed in [17] that help to manage network traffic demands and radio channel constraints. Efficient channel allocation can help to minimize traffic congestion in WMN. The CA schemes proposed in [18], [19] and [20] report to mitigate co-channel interference i.e., the same frequency between two nodes that are adjacent in multi-radio multi-channel networks. CA schemes based on conflict graphs are reported to contribute significantly towards improved channel utilization and thus reduce interference problem. The interference aware channel assignment algorithm proposed in [21], for multi-radio WMN, helps to assign channels to the radio that minimizes co-located interference.

In [22], an interference estimation and CA performance prediction technique is proposed for MRMC- WMN deployments, using conflict free graph approach [22]. CA scheme used for 
performance estimation is based on computational cost and algorithmic complexity. However, in this work, throughput based on individual link quality estimation is not considered.

A generic algorithm is proposed in [23] for conflict graph generation, which is used to identify and estimate interference in wireless networks. However, no algorithm is formulated based on channel assignment strategy for co-located radio interference problem. Where as the existing Breadth First Search Channel Assignment (BFS-CA) [11] and Maximal Independent Set Channel Assignment (MaIS-CA) [24] algorithm are compared for performance analysis [23].

Future wireless networking technologies, such as LTE-A and 5G, strive to improve provisioning of data services by considering QoS issues related to transmission delay, jitter, bandwidth, etc. [25] [26]. Multi-radio can be enabled in each node for simultaneous transmission and to improve network performance. However, in case of complex networks with dense deployment, this may also result in severe interference problems. Channel assignment, routing and scheduling [27] play a role in mitigating the interference (i.e. NP-Complete problem) to achieve minimal channel utilization.

The existing CA algorithms presented in [11] and [24] establish that optimal channel assignement which addressing radio based interference problem is NP-complete and therefore network optimality cannot be achieved. The broadband wireless networks often use OFDM to provide better QoS in WMN. Such wireless networks using OFDM have to enable multi-radios, to support simultaneous transmissions, in each network node. This can lead to occurrence of interference and thus deterioration in network performance. CA based strategies can help to mitigate interference related to multi-radios in WMN. The main objective of any CA strategy is to provide QoS with minimal number of channel utilization. In this study, we address the problem of multi-radios that are co-located and co-channel interference and minimal number of channels required for such network is determined to achieve improved Quality of Service (QoS) in WMN.

\section{Problem Definition}

For any MRMC network, such as the one represented in Fig. 1(a), the topology of the network can be represented as a Graph G $(V, E)$, where V denotes a set of vertices and E denotes the set of links between network nodes. Co-channel interference occurs when neighbouring or adjacent nodes use same channel. Let's assume that the nodes are enabled with radios ' $r_{n}$ ' where $\mathrm{E} 1$ to $N$. Then, $G$ $(\mathrm{V}, \mathrm{E})$ can be represented as, $\mathrm{G}\left(\mathrm{V}(\mathrm{i}) \mathrm{r}_{\mathrm{n}}, \mathrm{E}(\mathrm{i})\right)$, where ' $\mathrm{r}$ ' denotes radio and ' $\mathrm{n}$ ' is the number of radios enabled for any node.

$$
\begin{aligned}
& \text { Where, } V(i) r_{n}=\left\{\left(V(1) r_{1}, V(1) r_{2}, . .\right),\left(V(2) r_{1}, V(2) r_{2}, V(2) r_{3}, . .\right) \ldots . .\left(V(n) r_{1}, V(n) r_{2}, \ldots\right)\right\} \\
& E_{i}=\left\{E_{1}, E_{2}, \ldots E_{n}\right\} . \text { ' } i \text { ' \& ' } n \text { ' } C 1 \text { to infinity. }
\end{aligned}
$$




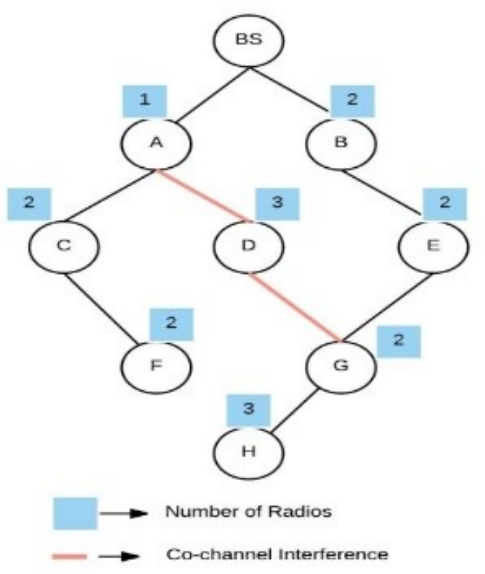

(a)

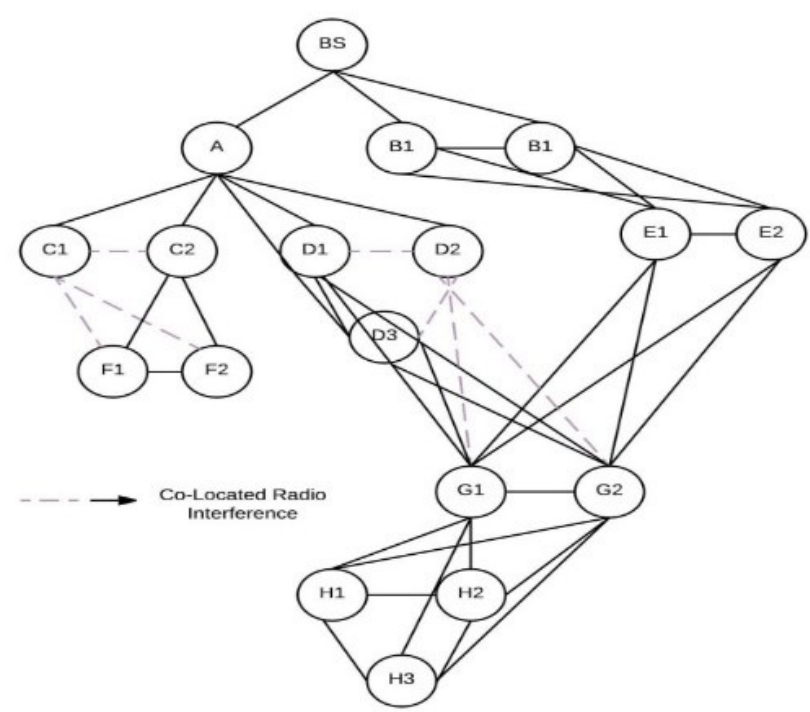

(b)

Fig. 1 a) Network topology b) Network topology with radios represented as nodes

A network node that is enabled with multiple radios, is represented here such that all its radios are linked with those of neighboring nodes, forming a fully connected graph. The linear MRMC network flows, equipped with multiple radios are represented in Fig. 1(a) and 1(b). This study assumes that nodes in $\mathrm{G}(\mathrm{V}(\mathrm{i}) \mathrm{r}, \mathrm{n}, \mathrm{E}(\mathrm{i}))$ are densely connected and co-located radio interference occurs between adjacent radios within a node. First, interference identification helps to classify conflicting links in the network by identifying interference. As far as QoS is concerned, assigning different channels does not contribute towards improvement in network performance. A two folded algorithm converts the unlying graph into a Line graph ' $\mathrm{L}$ ' as depicted in figure 2 . This is carried out to distinguish and classify conflicting and non-conflicting links, in order to avoid occurance of interference problem.
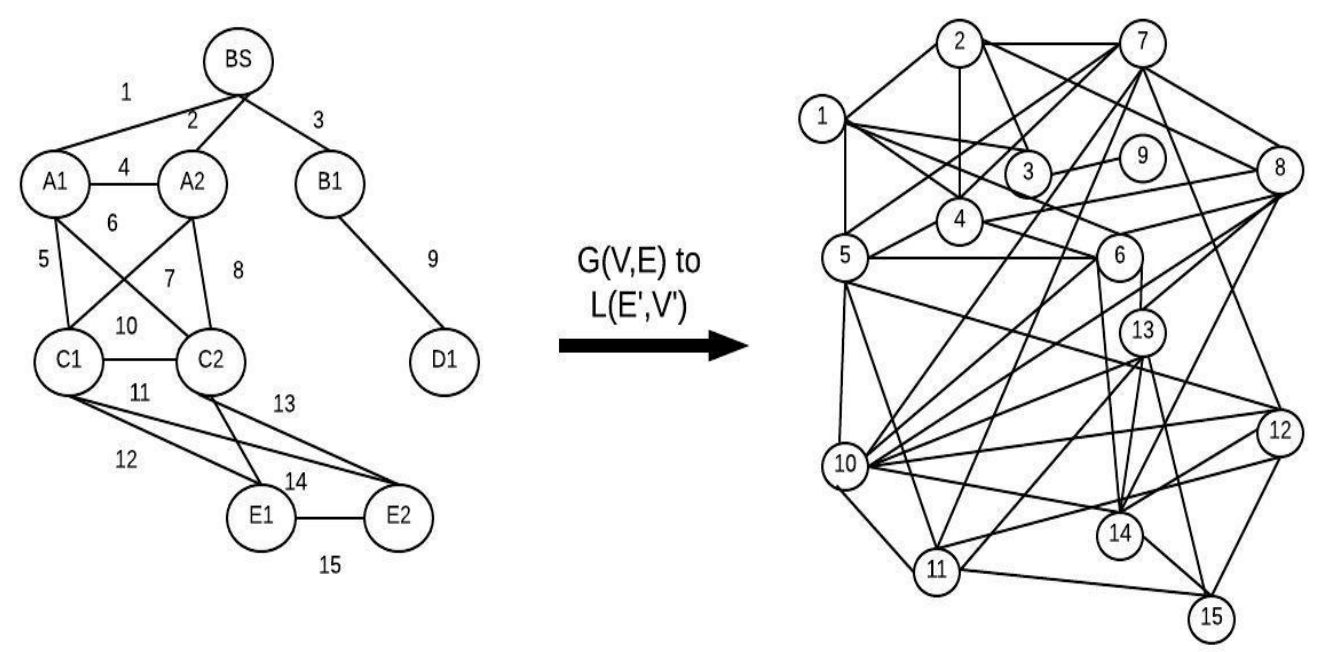

Fig. 2 Network into Line Graph L (G) 
The proposed algorithm then convert Line graph ' $\mathrm{L}$ ' into a Path graph ' $\mathrm{P}$ ' to obtain minimal number of channel utilized in the network by mitigating co-located radio interference. Sample path graph sequence of Two-Folded CA algorithm is presented in Fig. 3.
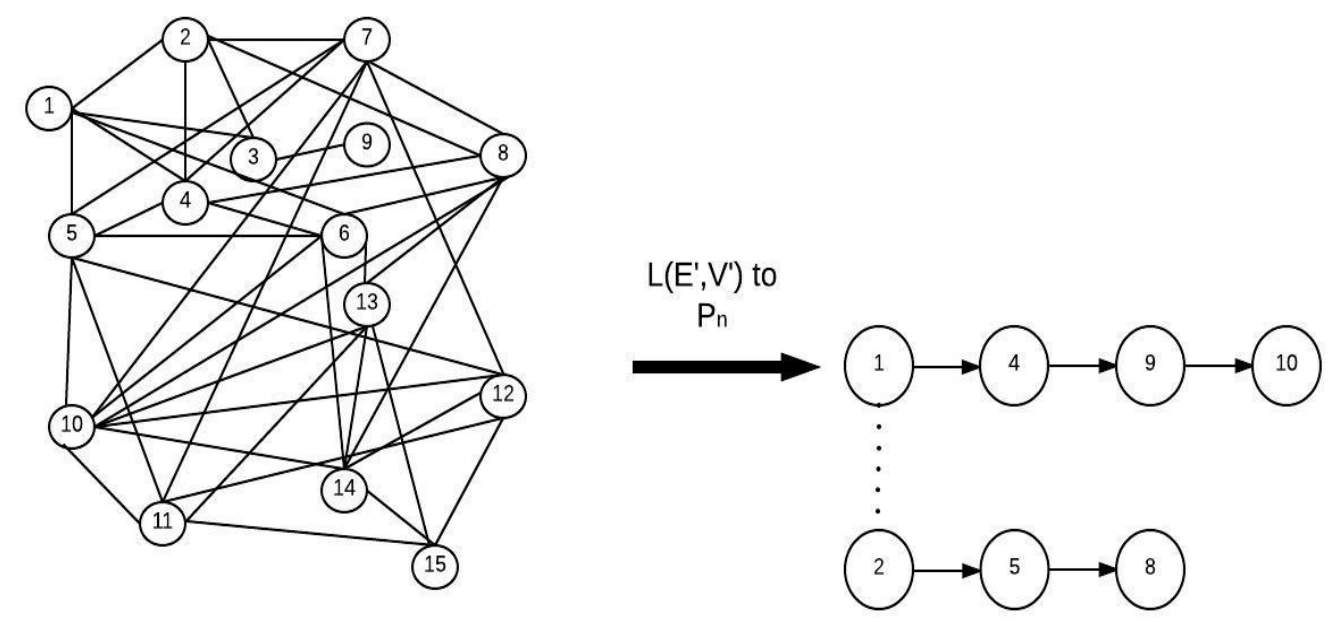

Fig. 3 Line Graph L(G) into Path Graph $P_{n}$ representation

\section{Proposed Ra-CA strategy}

In this section, we proposed Ra-CA strategy that solves the problem of co-channel and co-located radio interference to determine minimal number of channels required in MRMC network for effective QoS. The Ra-CA scheme is subdivided into two algorithms; independent set and twofolded algorithms, which are developed based on direct graphical method and the classification of interfering and non-interfering nodes. The independent set algorithm forms a non-conflict index, which are interference free links with different channel utilization. Two-folded algorithm helps to reduces network traffic and transmission delay, which resolves the co-located radio interference in MRMC networks.

\subsection{Interference node level $\left(m_{i}\right)$ :}

Interference node level $\left(m_{i}\right)[27,10]$ is defined here as the number of connected conflicting nodes to get interfering links and is represented in equation (1).

$$
m_{i}=\sum_{\mathrm{k}=1}^{\mathrm{n}} \mathrm{I}_{\mathrm{k}}
$$

Where, $\mathrm{k}$ is the connected neighbor nodes of a particular node ' $\mathrm{I}$ '. Degree sum $\left(D_{S}\right)$ is calculated for each transmission from source to destination as:

$$
\mathrm{D}_{\mathrm{s}}=\sum_{\mathrm{j}=0}^{\mathrm{k}}\left(\sum_{\mathrm{i}=0}^{\mathrm{n}} \mathrm{m}_{\mathrm{i}}\right)
$$

Where, i is the connected neighbouring nodes from 1 to $\mathrm{n} ; \mathrm{j}$ is the transmitting node from source to destination from 1 to $\mathrm{k}$.

The interference node levels help to schedule link transmission events to be processed, as nodes are enabled with multiple radios. The interference graph for the particular network is then generated to classify neighbouring and non-neighbouring links. 


\subsection{Independent set algorithm:}

Independent set algorithm helps to classify interfering and non-interfering links by identify the colocated radio interference in the network. With the line graph ' $\mathrm{L}$ ', minimal interfering node Ds is selected. Then each vertex in $E_{n}$, which is non-neighbouring set of Ds, is chosen and minimum degree vertex is sorted out from the list $\mathrm{E}_{\mathrm{n}}$. Again the minimum interfering node Ds is selected so that the non-adjacent vertices in $E_{n}$ are listed. This procedure is repeated till all Ds elements in the network are processed.

The selected vertex of Ds forms a non-conflict index that is feasible and interference free. All the vertices of ' $L$ ' are selected and several index sets are formed. These non-conflict index sets help to avoid both co-located radio and co-channel interference with free assignment of a channel to the links in the network. Considering $v \in L(G)$ and $d\left(E_{1}\right)$ and $d\left(E_{2}\right)$ are the two vertices having same minimum degree, then choose any one $\left[d\left(E_{1}\right) \| d\left(E_{2}\right)\right]$ such that it may not create any impact.

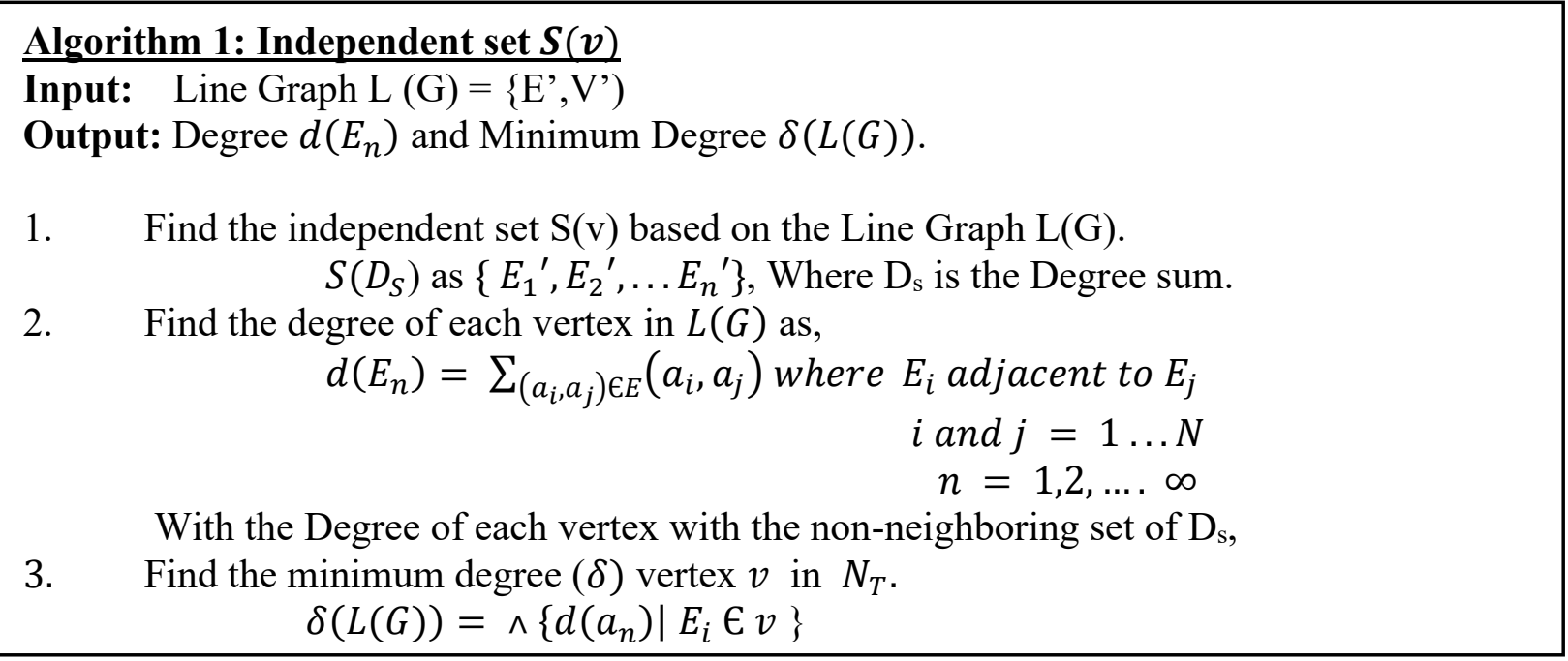

\subsection{Two folded CA algorithm:}

In two folded algorithm, one fold decides non-conflict index sets and in the other one non-conflict index sets are represented as Path graph ' $\mathrm{P}_{\mathrm{n}}$ '. For each ' $\mathrm{P}_{\mathrm{n}}$ ', the independent set $\mathrm{S}(\mathrm{v})$ algorithm is repeated for $\mathrm{CA}$ index sets, which provide a minimal channel utilization to the particular network.

The graphical conversion of the topological graph ' $G$ ' to ' $L$ ' and then subsequently to ' $\mathrm{P}_{\mathrm{n}}$ ', helps to mitigate co-located radio and co-channel interference problem in WMN. Path graph ' $\mathrm{P}_{\mathrm{n}}$ ' index sets provide minimal number of channels needed for WMN to provide better QoS. 


\section{Algorithm 2: Two-Folded CA Algorithm:}

Input: Independent Set S(v)

Output: Index with number of channels

1. With the Independent Set $\mathrm{S}(\mathrm{v})$, find the degree $d\left(E_{n}\right)$ and Minimum Degree $\delta(L(G))$ till it gets NULL.

2. Then sequence based on selected $d\left(E_{n}\right)$ and $\delta(L(G))$ is computed and denoted as non-conflict Index $I_{V}=\left\{I_{V 1}, I_{V 2}, \ldots I_{V n}\right\}$ where $n=1,2 \ldots \infty ; I_{V n}=\left\{e_{1}, e_{2}, \ldots e_{n}\right\}$.

3. With each $I_{V}$, a Path graph $P_{n}$ is computed where $\mathrm{n}=1,2 \ldots \mathrm{N}$.

4. $\quad$ Repeat Step 1 to get sequence based on selected $d\left(E_{n}\right)$ and $\delta(L(G))$ denoted as CA Index $I_{C}=\left\{I_{C 1}, I_{C 2}, \ldots I_{C n}\right\}$ where $n=1,2 \ldots \infty ; I_{C n}=\left\{e_{1}, e_{2}, \ldots e_{n}\right\}$

\section{Performance Analysis}

The following network configurations, presented in table 1, are assumed for performance analysis of the proposed Ra-CA scheme.

Table. 1 Network Configuration

\begin{tabular}{|l|l|}
\hline \multicolumn{1}{|c|}{ Parameter } & \multicolumn{1}{c|}{ Value } \\
\hline Modulation Type & OFDM_QPSK_1_2 \\
\hline Transmission Coverage & $500 \mathrm{~m}$ \\
\hline Network Interface Type & WirelessPhy/OFDMA \\
\hline Antenna & Omni Antenna \\
\hline Routing & DSDV \\
\hline Interface Queue Type & PriQueue \\
\hline Schedule Type & Wimax Scheduler \\
\hline Scheduling Flow & $\begin{array}{l}\text { rtPS_SCHEDULING } \\
\text { BE_SCHEDULING }\end{array}$ \\
\hline Packet Size & 1000 bits \\
\hline Agent & PT_WIMAXBS \\
\hline Seed RNG & HEURISTIC_SEED_SOURCE \\
\hline UlBurst & UIUC_FAST_RANGING \\
\hline DlBurst & DIUC_PROFILE [1 -11$]$ \\
\hline
\end{tabular}

A random topology is considered where a total of 30 nodes are simulated and analysed. The transmission range is defined as $250 \mathrm{~m}$ and the number of radios varies from 1 to 5 per node using 
ns-2 simulation. A central controller BS is created along with subordinate SS's where the number of sufficient or insufficient channels has an impact on the multi-radio network. In the case of centralized scheduling, BS takes control as a centralized entity and all activities of SS's are maintained and monitored by it. In this scheduling mechanism, CA strategy is provided that is responsible for utilizing minimal number of channels. Based on the number of channel usage, following cases could occur.

\section{Case (a): Sufficient number of channels:}

A minimal number of channel utilization in the network is provided by avoiding co-channel and co-located radio interferences, where channel utilization is calculated and throughput optimization is considered. Here an MRMC network is assumed, where links can be assigned a minimal number of channels. With the existence of a sufficient number of channels, an appropriate channel allocation scheme could be employed to eliminate interference entirely, by avoiding co-locate radio inferences. There are three types of methods such as direct, induction and contemporary methods [31] [32]. Where the induction method examines that the sufficient number of channels condition is true. The deduction method examines that the sufficient number of channels condition is false. Direct method generalizes the condition of sufficient number of channels.

For this kind of network, the following case (a) is proven with the method of induction as,

$$
(a+b)^{T}=a^{T}+b^{T} \text { where } n=1,2, \ldots N .
$$

Here ' $\mathrm{T}$ ' is considered as time, and then all the packets first exist in the base station $\mathrm{BS}$ at $\mathrm{T}=0$. At $\mathrm{n}=1$, then induction method as $(a+b)^{1}=a^{1}+b^{1}$ then the packets move to the level 1 and the packets to level 2 as,

$$
\begin{aligned}
(a+b)^{2}= & a^{2}+b^{2}+2 a b \text { where } \mathrm{ab} \text { and ba have interference as NULL } \\
& (a+b)^{2}=a^{2}+b^{2} \& \&(a+b)^{3}=a^{3}+b^{3}
\end{aligned}
$$

Based on level 3, hop count three is assigned as the highest for all the packets between the source and destination. At time $\mathrm{T}=\mathrm{k}$, with the sufficient number of channels, the hop count is created to be 2 and also for $\mathrm{T}=\mathrm{k}+1$ based on the induction method. Two packets with the levels $\mathrm{T}$ and $\mathrm{T}-3$ at $\mathrm{T}=\mathrm{k}+1$ are considered; then the difference is 1 for hop count. So level ' $\mathrm{i}$ ' is selected for transmission, which has no interference while $\mathrm{T}=\mathrm{k}$ has hop count difference of 2 . Based on the induction method,

$$
(a+b)^{k}=a^{k}+b^{k} \& \&(a+b)^{k+1}=a^{k+1}+b^{k+1}
$$

This case is valid for all the channels and applied to all the networks.

\section{Case (b): Transmitter / Receiver based interference free channels}

[39] explains the contemporary methods if the condition is proved to be false for the sufficient number of channels. Based on the contemporary method, this case is false for this network because this network supports multi-radio transceiver i.e. interference avoidance is negligible for further co-channel avoidance. So, the function $f(T)$ and $f(R)$ where Transmitter ' $T$ ' and Receiver ' $R$ ' are neglected.

\section{Case (c): Insufficient number of channels}

If the condition of insufficient number of channels is true as in [39], CA algorithm is used to avoid 
interference. With the Graph $\mathrm{G}(\mathrm{E}, \mathrm{V})$, a Line graph $\mathrm{L}(\mathrm{V}, \mathrm{E})$ is first constructed. Then, independent subsets are identified based on the min-max degree of a node and hop count for interference avoidance to assign channels in the network. With the interference results, Path graph $\mathrm{P}_{\mathrm{n}}$ is then created from $\mathrm{L}(\mathrm{V}, \mathrm{E})$ to achieve subset for interference avoidance and to provide a minimal number of channels in the network. This procedure makes the decision to carry out this case for an insufficient number of channels.

\subsection{Variation in the number of channels with different QoS parameters}

In this section, we have compared proposed Ra-CA with the existing algorithms namely, BFS-CA [19] and MaIS-CA [20]. With the channel variation, the Probability Delivery Ratio (PDR) is calculated from equation (3) [28]. The proposed Ra-CA has more marginal increase than the existing one for all the variations up to 5 channels. The proposed Ra-CA and the existing BFS-CA have a slight variation, as an increase in the number of channels is represented in Fig. 4(a).

$$
\text { Probability Delivery Ratio }(\mathrm{PDR})=\frac{\text { Number of packets received }}{\text { Number of packets sent }}
$$

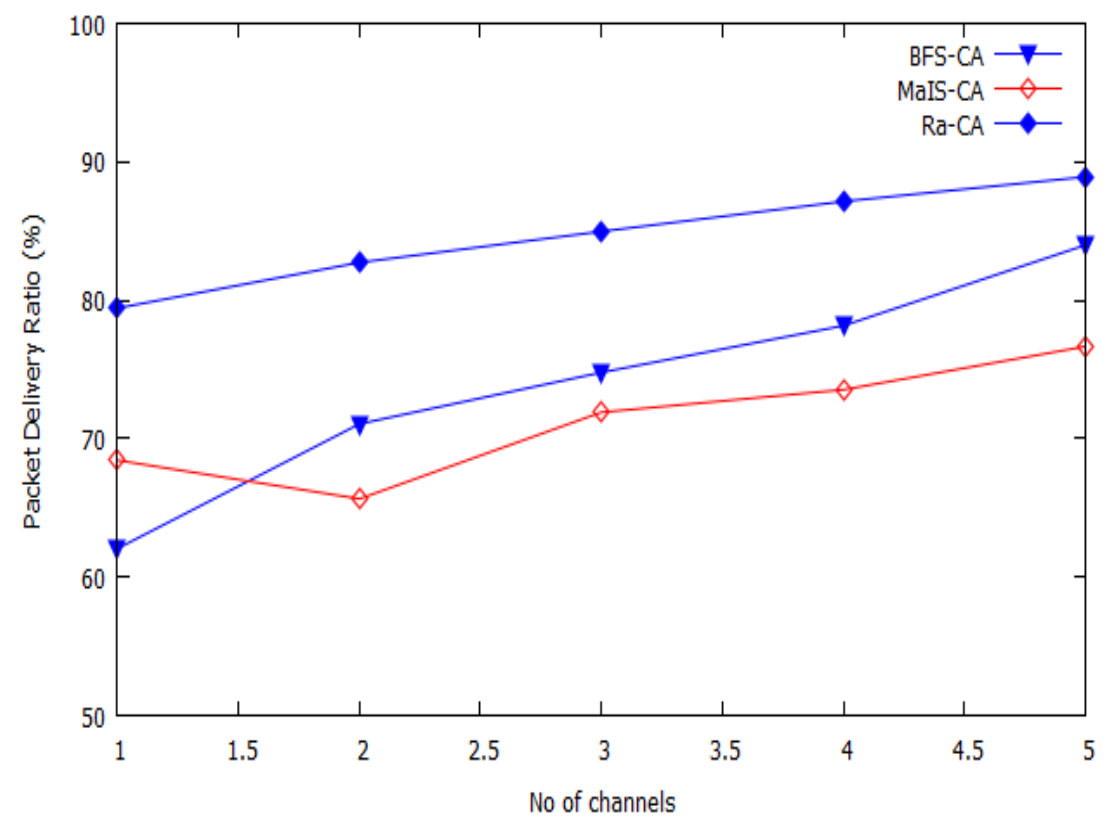

Fig. 4 (a) Packet Delivery Ratio Vs Number of Channels 


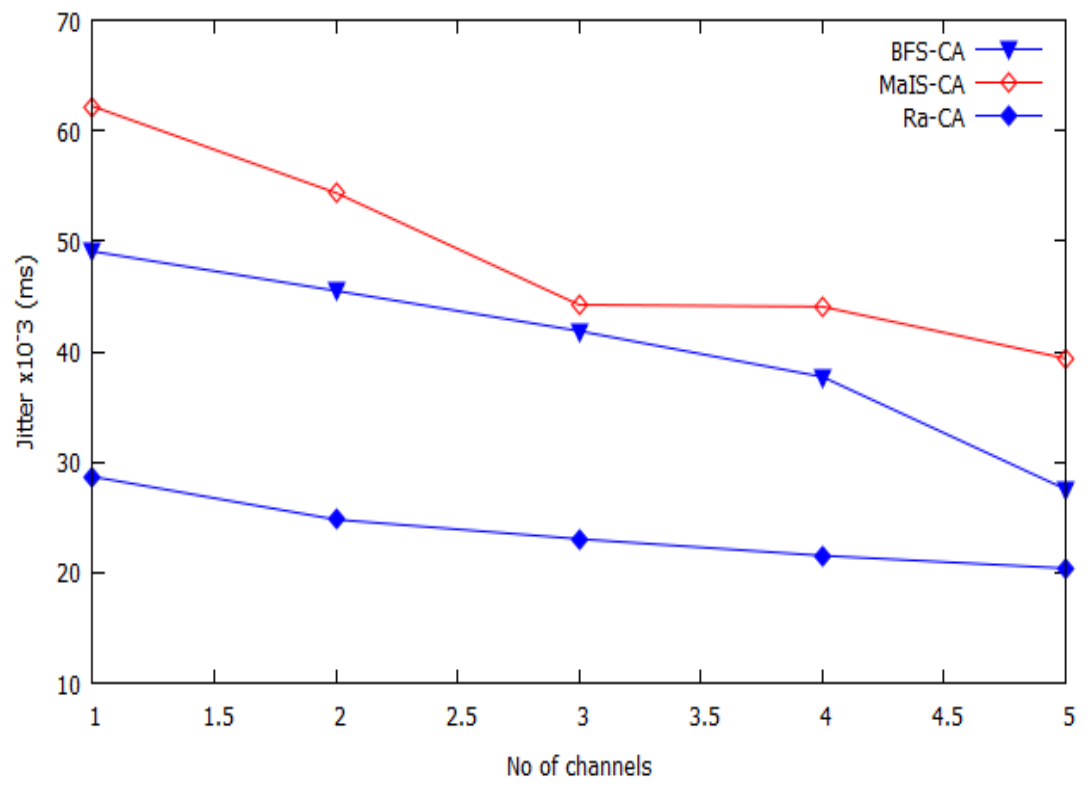

Fig. 4 (b) Jitter Vs Number of Channels

When the sender side transmits packets, any variation in the delay of received packets is considered as jitter and is given in equation (4) [28]. Based on the determination of notation used in equation (3) [29] for PDR, the proposed Ra-CA decrease time interval at the destination, when compared to the existing algorithms represented in Fig. 4(b).

Jitter $=$ Actual Transmission Time - Expected Transmission Time

In case of the proposed Ra-CA scheme, delay is calculated for varying number of channels and is shown in Fig. 5(a). As the number of channels range from 1 to 5, the delay decreases with 30 nodes being analysed. The transmission delay decreases gradually with the minimal number of channels limit. However, as the number of channels get increased with over channel usage, the transmission delay seem not to maintain this gradual decrease. The transmission delay, given by equation (5), is calculated based on the number of packets sent with the starting time and finishing time. 


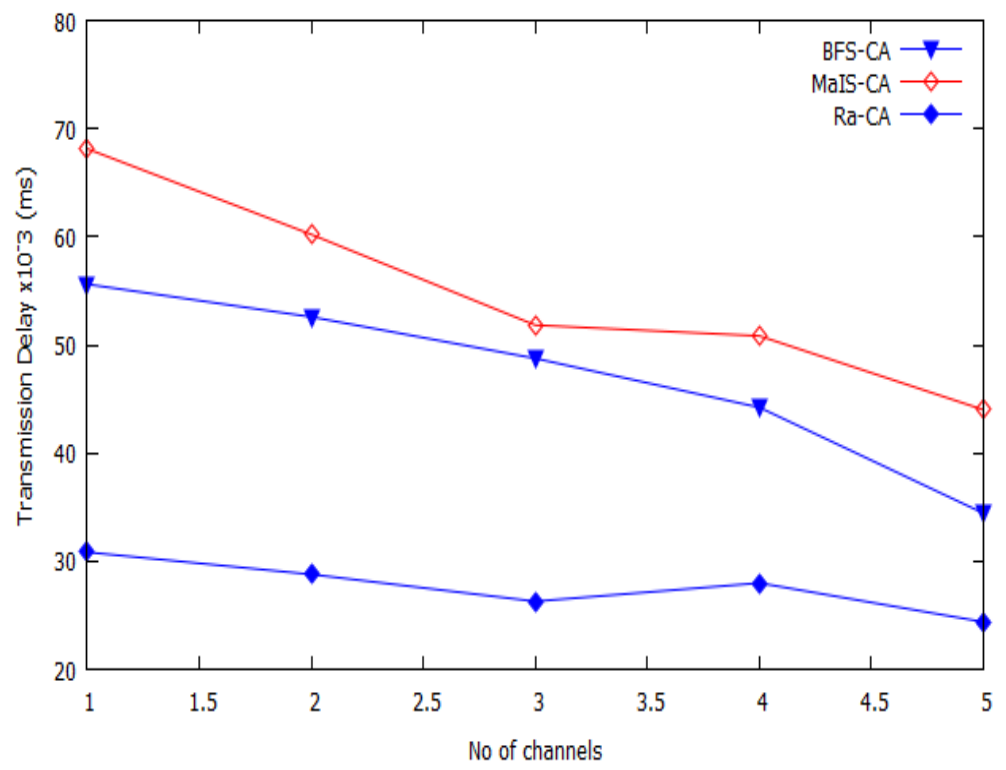

Fig. 5 (a) Transmission Delay Vs Number of Channels

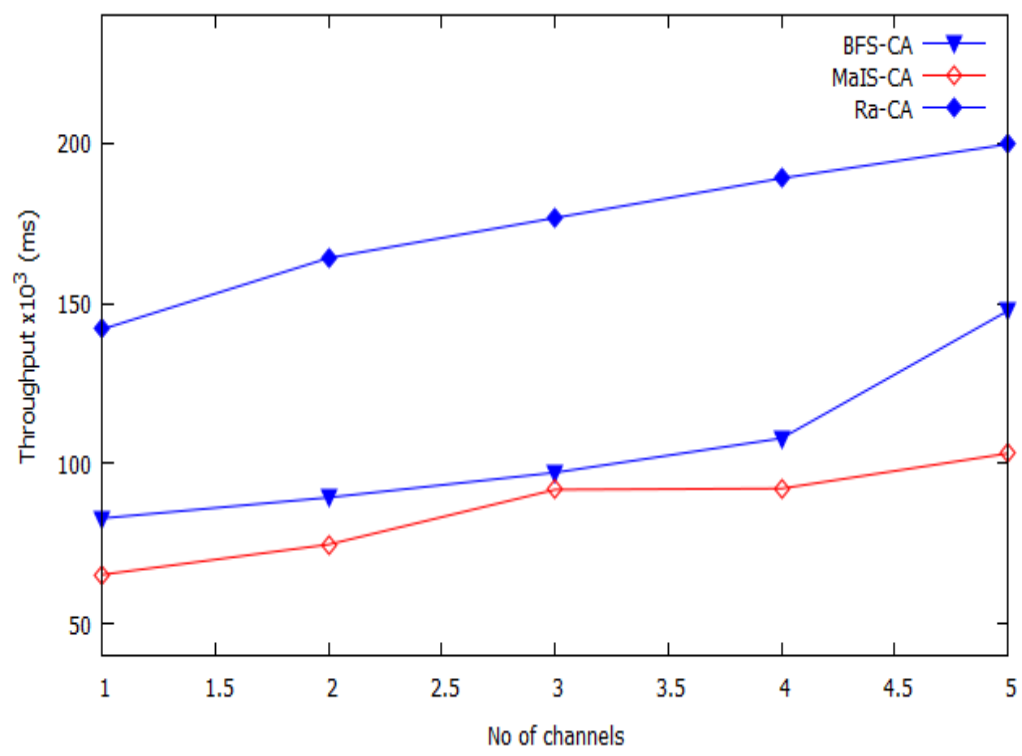

Fig. 5 (b) Throughput Vs Number of Channels

Transmission Delay $=\frac{\text { (FinishedTime-StartingTime })}{\text { Number of packets sent }}$

In Fig. 5(b), BFS-CA and MaIS-CA have little difference based on the throughput of the network. However, the proposed Ra-CA scheme has a gradual increase in the throughput based on the variation in the number of channels, and is given by equation (6) [30]. For the same delay parameter, as in Fig 5(a), throughput also increase with the exact channel utilization count. The channel gets increased, but the throughput maintains its stable level in Fig. 5(b). 


$$
\text { Throughput }=\frac{\text { Number of packets recieved } * 8 * 512}{((\text { FinishedTime-StartingTime }) * \text { Probability delivery ratio })}
$$

\subsection{Comparison of the proposed strategy with heterogeneous networks scenario}

The proposed Ra-CA strategy is implemented with configuations as discussed in Table. 1. In Fig. 6(a) and 6(b), the proposed Ra-CA strategy is also implemented with WiMAX + LTE as heterogeneous networks. In this heterogeneous network, the network is provided with several LTE agents like dmagent, enbagent, lteagent, mmeagent, ulslagent, etc. to analyse the performance based on delay and throughput. Simulation time is the time required to complete the individual process or task in an estimated time.

In Fig. 6(a), delay gets decreased in Ra-CA (WiMAX + LTE) when compared to Ra-CA (WiMAX) based on the variation in the simulation time. In Fig. 6(b), throughput gets increased in Ra-CA (WiMAX + LTE) when compared to proposed Ra-CA (WiMAX) with the difference in the simulation time.

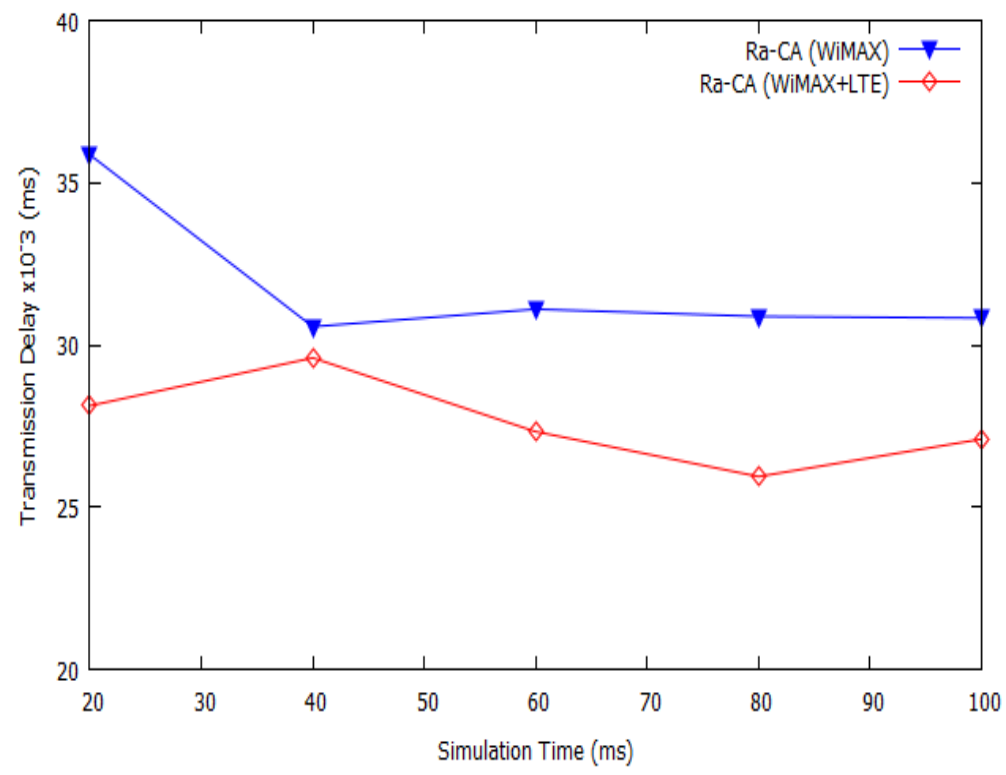

Fig. 6 (a) Comparison of the Number of Channels with the Transmission Delay 


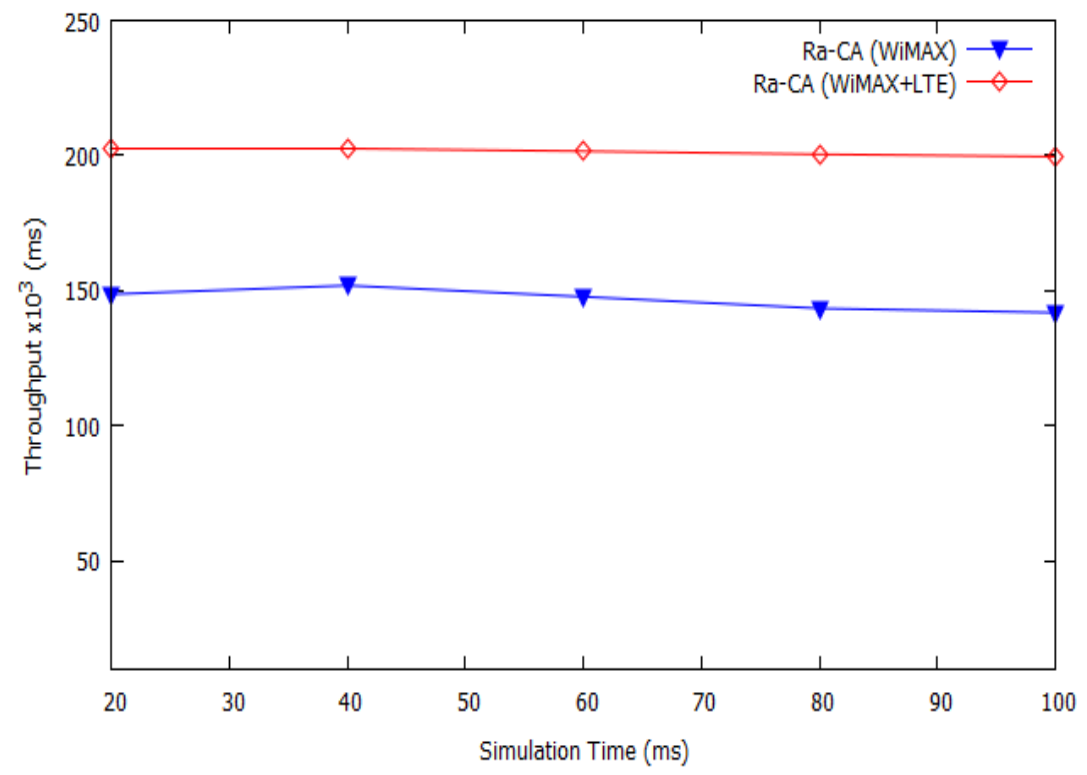

Fig. 6 (b) Comparison of the Simulation Time with the Throughput

\subsection{Effect of QoS based on the simulation time}

In this section, the QoS parameter is analyzed for varying simulation time against channel usage count of 1 and 5. The channel count is fixed for the proposed Ra-CA and is compared with the existing BFS-CA and MaIS-CA

As shown in Fig. 7(a), the transmission delay reaches a steady level as simulation time increases. At the initial simulation time $20 \mathrm{~ms}$, the proposed Ra-CA gets little variation compared to the existing algorithms, but as the simulation time increased, delay variation gets increased with the existing BFS-CA and MaIS-CA algorithm.

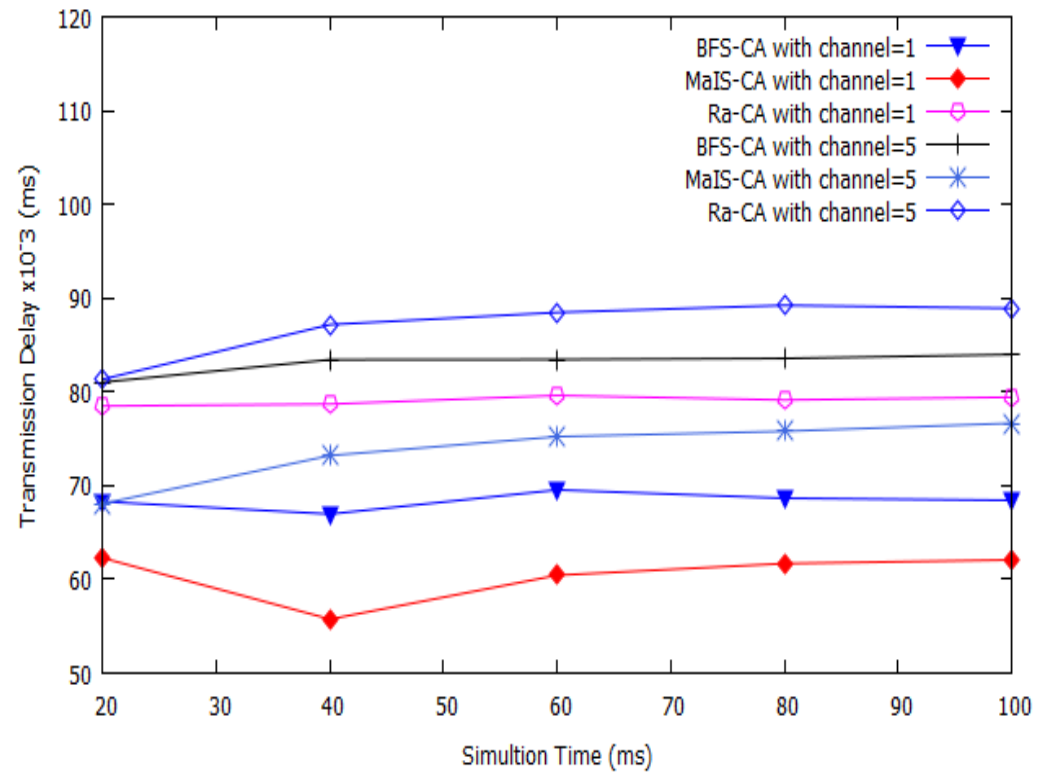

Fig. 7 (a) Simulation Time Vs Transmission Delay 


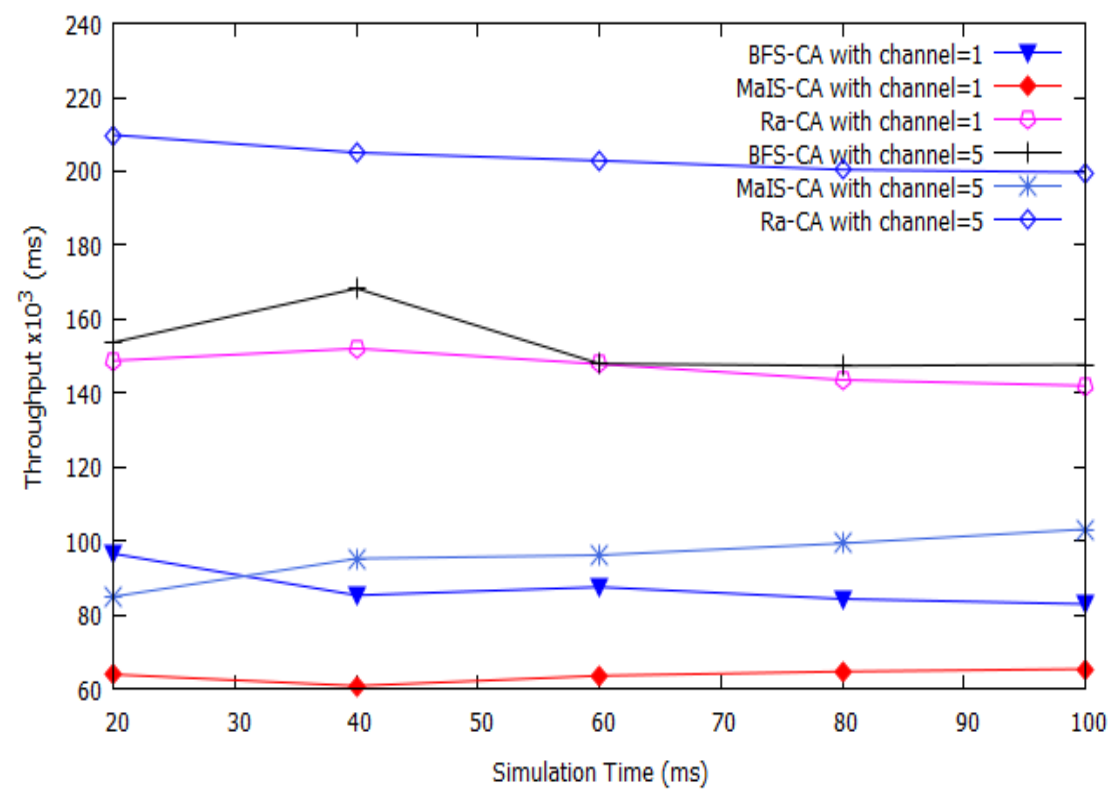

Fig. 7 (b) Simulation Time Vs Throughput

With the channel count of 5, the existing BFS-CA and MaIS-CA have a slight variation based on the simulation time, but the proposed Ra-CA has increased throughout with constant level with the simulation time difference. As same as in Fig. 7(a), throughput also increased with the number of channel usage count as in Fig. 7(b).

On the base of these simulation results, we can clearly claim that proposed Ra-CA is better than existing algorithms namely BFS-CA and MaIS-CA in terms of QoS parameters such as, packet delivery ratio, packet drop, transmission delay and throughput. This work can considerably benefit advanced networks like LTE and hetrogenous wireless networks.

\section{Conclusion}

Based on CA strategy, the Ra-CA algorithm is proposed in a multi-radio multi-channel mesh network. Our strategy involves a minimal number of channels with the avoidance of both colocated radio and co-channel interference when compared to the existing algorithms such as BFS$\mathrm{CA}$ and MaIS-CA. In the simulation, the effect of QoS parameters based on channel variation is studied which helps to analyse the performance of Ra-CA algorithm. The variation in the number of channels is observed for the proposed Ra-CA with the increased amount of QoS services i.e. packet delivery ratio, packet drop, transmission delay and throughput. Ra-CA strategy is compared with the number of channel usage with the avoidance of co-located radio interference. Compared to the existing schemes, the proposed algorithm provides a radio based interference aware resource allocation with increasing network throughput. The proposed algorithm supports uplink/downlink. In the future study, this problem can be addressed in Next Generation Networks such as 5G Heterogeneous Networks (HetNets) as it provides multi-radio concept i.e. OFDM and performance analysis and future directed towards the distributed scheduling are to be considered 


\section{Acknowledgements}

Ramkumar Jayaraman gratefully acknowledges support from Anna University - Anna Centenary Research Fellowship. Gunasekaran Raja, Ramkumar Jayaraman gratefully acknowledges support from NGNLabs, Department of Computer Technology, Anna University, Chennai.

\section{REFERENCES}

1. http://mobilebusinessinsights.com/2016/07/wireless-mesh-networking-improves-connectivity-inenter prise-ecosystems/

2. Djohara Benyamina, Abdelhakim Hafid and Michel Gendreau, "Wireless Mesh Networks Design — A Survey,” IEEE Communications Surveys \& Tutorials, Vol. 14, Issue. 2, pp. 299 - 310, Second Quarter 2012.

3. Syed Yasmeen Shahdad, Asfia Sabahath and Reshma Parveez, "Architecture, issues and challenges of wireless mesh network," in Proc. International Conference on Communication and Signal Processing (ICCSP), pp. 557 - 560, April. 2016.

4. Najah Abu-Ali, Abd-Elhamid M. Taha, Mohamed Salah and Hossam Hassanein, "Uplink Scheduling in LTE and LTE-Advanced: Tutorial, Survey and Evaluation Framework," IEEE Communications Surveys \& Tutorials, Vol. 16, Issue 3, pp. 1239 - 1265, Third Quarter 2014.

5. Ahmad Mudassir, Saleem Akhtar and Hesham Kamel, "Survey on inter-cell interference coordination in LTE-Advanced heterogeneous networks," Sixth International Conference on Innovative Computing Technology (INTECH), pp. 429 - 434, Aug. 2016.

6. Saumitra M. Das, Dimitrios Koutsonikolas, Y. Charlie Hu and Dimitrios Peroulis, "Characterizing multi- way interference in wireless mesh networks," In proceedings of the 1st international workshop on Wireless network testbeds, experimental evaluation \& characterization, WiNTECH '06, pp. $57-64$, Sept. 2006.

7. Sunghun Kim, Jongsub Cha and Joongsoo Ma, "Interference-Aware Channel Assignment with Seamless Multi-Channel Monitoring on Wireless Mesh Network," In proceedings of 6th IEEE Consumer Communications and Networking Conference, CCNC 2009, DOI: 10.1109/CCNC.2009.4784793, Feb. 2009.

8. S. Kim, J. Cha and J. Ma, "Interference-Aware Channel Assignments with Seamless Multi-Channel Monitoring in Wireless Mesh Networks," IEEE International Conference on Communications, ICC '09, DOI: 10.1109/ICC.2009.5199095, Aug. 2009.

9. Yuhuai Peng, Yao Yu., Lei Guo, Dingde Jiang and Qiming Gai, “An efficient joint channel assignment and QoS routing protocol for IEEE 802.11 multi-radio multi-channel wireless mesh networks," Journal of Network and Computer Applications, Elsevier Publications, Vol. 36, Issue. 2, pp. 843 - 857, Mar. 2013.

10. Ramkumar Jayaraman, Gunasekaran Raja, Dipak Ghosal, Rajakumar Arul and Sabareesh Kumar A, "A Compatibility Vector Technique for Cooperative Scheduling and Channel Assignment Algorithm in Broadband Wireless Networks" in Springer - Journal of Mobile Networks and Applications (MONETS), Mar. 2017, Doi: 10.1007/s11036-017-0841-x.

11. K. N. Ramachandran, E. M. Belding, K. C. Almeroth and M. M. Buddhikot, "Interference-Aware Channel Assignment in Multi-Radio Wireless Mesh Networks," in Proc. 25th IEEE International Conference on Computer Communications, INFOCOM, 2006, pp. 1 - 12, April. 2006. 
12. A. H. M. Rad and V. W. S. Wong, "Joint Channel Allocation, Interface Assignment and MAC Design for Multi-Channel Wireless Mesh Networks," In proceedings of 26th IEEE International Conference on Computer Communications, INFOCOM 2007, DOI: 10.1109/INFCOM.2007.173, May 2007.

13. Kun Xie, XinWang, Xueli Liu, Jigang Wen, and Jiannong Cao, Interference-Aware Cooperative Communication in Multi-Radio Multi-Channel Wireless Networks, IEEE Transactions On Computers, Vol. 65, No. 5, pp. 1528 - 1542, May 2016.

14. Hung-Yu Wei, S. Ganguly, R. Izmailov and Z.J. Haas, "Interference-aware IEEE 802.16 WiMax mesh networks," 2005 IEEE $61^{\text {st }}$ Vehicular Technology Conference, VTC 2005-Spring, pp. 3102 3106, Dec. 2005.

15. Wen-Hwa Liao, Sital Prasad Kedia and Avinash Kumar Dubey, "Scheduling and channel assignment algorithm for IEEE 802.16 mesh networks using clique partitioning technique," Journal of Computer Communications, Vol. 35, Issue 16, pp. 2025-2034, May 2012.

16. Chieochan, S. and Hossain, E, "Channel Assignment for Throughput Optimization in Multichannel Multiradio Wireless Mesh Networks Using Network Coding," IEEE Transaction on Mobile Computing, Vol. 12, Issue. 1, pp. 118 - 135, Jan. 2013.

17. Jonathan Wellons and Yuan Xue, " The robust joint solution for channel assignment and routing for wireless mesh networks with time partitioning," Ad Hoc Networks, Elsevier Publications, Vol. 13, Part A, pp. 210 - 221, Feb. 2014.

18. A. B. M. Alim Al Islam, Md. Jahidul Islam, Novia Nurain and Vijay Raghunathanm, "Channel Assignment Techniques for Multi-Radio Wireless Mesh Networks: A Survey," IEEE Communications Surveys \& Tutorials, Vol. 18, Issue. 2, pp. 988 - 1017, Second Quarter 2016.

19. Mohammad Doraghinejad, Hossein Nezamabadi-pour and Ali Mahani, "Channel assignment in multi-radio wireless mesh networks using an improved gravitational search algorithm," Journal of Network and Computer Applications, Elsevier Publications, Vol. 38, pp. 163 - 171, Feb. 2014.

20. Jihong Wang, Wenxiao Shi, Keqiang Cui, Feng Jin and Yuxin Li, "Partially Overlapped channel assignment for multi-channel multi-radio wireless mesh networks," EURASIP Wireless Communication and Networking, Springer, Feb. 2015, doi: 10.1186/s13638-015-0259-8.

21. Jian Tang, Guoliang Xue and Weiyi Zhang, "Interference-aware topology control and QoS routing in multi-channel wireless mesh networks," in Proc. ACM international symposium on Mobile ad hoc networking and computing, MobiHoc '05, pp. 68 - 77, May 2005.

22. Srikant Manas Kala, M. Pavan Kumar Reddy, Ranadheer Musham and Bheemarjuna Reddy Tamma, "Reliable prediction of channel assignment performance in wireless mesh networks," in Proc. International Conference on Advances in Computing, Communications and Informatics (ICACCI), pp. 13 - 19, Aug. 2015.

23. Srikant Manas Kala, M. Pavan Kumar Reddy, Ranadheer Musham and Bheemarjuna Reddy Tamma, "Interference mitigation in wireless mesh networks through radio co-location aware conflict graphs," Wireless Networks, Springer, Vol. 22, Issue. 2, pp. 679 - 702, Feb. 2016.

24. Aizaz U. Chaudhry, John W. Chinneck and Roshdy H. M. Hafez, "Channel Requirements for Interference-Free Wireless Mesh Networks to Achieve Maximum Throughput," in Proc. 22nd International Conference on Computer Communications and Networks (ICCCN), pp. 1 - 7, Oct. 2013. 
25. Singhal, Chetna and De, Swades, "Resource Allocation in Next-Generation Broadband Wireless Access Networks," Advances in Wireless Technologies and Telecommunication (AWTT), Book Series, 2017.

26. Hassen A. Mogaibel, Mohamed Othman, Shamala Subramaniam and Nor Asilah Wati Abdul Hamid, "Review of channel assignment approaches in multi-radio multi-channel wireless mesh network," Journal of Network and Computer Applications, Elsevier Publications, Vol. 72, pp. 113 - 139, Sept. 2016.

27. Wang Jihong, Shi Wenxiao and Jin Feng, "On channel assignment for multicast in multi-radio multi-channel wireless mesh networks: A survey," China Communications, IEEE, Vol. 12, Issue. 1, pp. 122 - 135, Jan. 2015.

28. Anand Prabhu Subramanian, Himanshu Gupta and Samir R. Das, "Minimum Interference Channel Assignment in Multi-Radio Wireless Mesh Networks," in Proc. 4th Annual IEEE Communications Society Conference on Sensor, Mesh and Ad Hoc Communications and Networks, SECON '07, pp. 481 - 490, Aug. 2007.

29. Deepali Ramesh Borade and Shaikh. Mohd. Laeeq, "Performance and evaluation of IEEE 802.15.4 under different topologies with Ad-hoc on demand distance vector protocol," in Proc. IEEE Students' Conference on Electrical, Electronics and Computer Science (SCEECS), Mar. 2012, Doi: 10.1109/SCEECS.2012.6184752.

30. http://disc.ece.illinois.edu/downloads/slabc1.html.

31. Biskup, M., Bovier, A., den Hollander, F., Ioffe, D., Martinelli, F., Netocný, K. and Toninelli, C., "Methods of Contemporary Mathematical Statistical Physics," Lecture Notes in Mathematics, Springer, 2009.

32. Irving Rothchild, "Induction, Deduction and the Scientific Method - An Eclectic Overview Of The Practice Of Science," Society for the Study of Reproduction, Inc, 2006.

33. Marina, M, K \& Das, S, R 2005, 'A topology control approach for utilizing multiple channels in multi-radio wireless mesh networks', in proceedings of IEEE BroadNets, pp. 381-390.

34. Murali Kodialam \& Thyaga Nandagopal 2005, 'Characterizing achievable rates in multi-hop wireless mesh networks with orthogonal channels', IEEE/ACM Transaction on Networking, Vol. 13 , issue. 4 , pp. $868-880$

35. Akyildiz, I, F \& Xudong Wang 2005, 'A Survey on wireless mesh networks', IEEE Communication Magazine, vol. 43, issue 9, pp. S23-S30.

36. Weisheng Si, Selvadurai Selvakennedy \& Albert YZ 2010, 'An overview of Channel Assignment methods for multi-radio multi-channel wireless mesh networks', Journal of parallel and Distributed Computing, Elsevier Publications, vol. 70, no. 5, pp. 505 - 524.

37. Michalak, M and Torsten Braun 2005, 'Common Gateway Architecture for Mobile Ad-Hoc Networks', In Proceedings of the Second Annual Conference on Wireless On-demand Network Systems and Services, WONS '05, pp. $70-75$.

38. Du, P, Jia, W, Huang, L \& Lu, W 2007, 'Centralized scheduling and channel assignment in multichannel single-transceiver wimax mesh network', In Proceedings of Wireless Communications and Networking Conference, WCNC, pp. 1734-1739. 